\title{
Synchrony Dynamics Across Brain Structures in Limbic Epilepsy Vary Between Initiation and Termination Phases of Seizures
}

\author{
Tiwalade Sobayo, Ananda S. Fine, Elizabeth Gunnar, Christine Kazlauskas, David Nicholls, \\ and David J. Mogul*, Member, IEEE
}

\begin{abstract}
Neuronal populations in the brain achieve levels of synchronous electrophysiological activity during both normal brain function and pathological states such as epileptic seizures. Understanding how the dynamics of neuronal oscillators in the brain evolve from normal to diseased states is a critical component toward decoding such complex behaviors. In this study, we sought to develop a more in-depth understanding of multisite dynamics underlying seizure evolution in limbic epilepsy by analyzing oscillators in recordings of local field potentials from three brain structures (bilateral hippocampi and anteromedial thalamus) in a kainic acid in vivo rat model of temporal lobe epilepsy extracted using the empirical mode decomposition (EMD) technique. EMD provides an adaptive nonlinear decomposition into a set of finite oscillatory components. Oscillator frequencies, power, and phase synchrony were assessed within and between sites as seizures evolved. Consistent patterns of low-frequency $(\sim 35 \mathrm{~Hz})$ synchrony occurred transiently during early-stage ictogenesis between thalamus and both hippocampi; in contrast, higher frequency $(\sim 120 \mathrm{~Hz})$ synchrony appeared between thalamus and focal hippocampus as seizures naturally terminated. These multi-site synchrony events may provide a key insight into how synchrony disruption via stimulation could be targeted as well as contribute to a better understanding of how brain synchrony evolves in epilepsy.
\end{abstract}

Index Terms-Coherence, empirical mode decomposition (EMD), epilepsy, temporal lobe.

\section{INTRODUCTION}

$\mathbf{E}$ PILEPSY is a brain disorder characterized by recurrent and spontaneous aberrant electrophysiological behavior in the brain, called epileptic seizures [1]. More than 50 million people worldwide have epilepsy. Because significant increases in the

Manuscript received September 26, 2011; revised December 16, 2011; accepted February 6, 2012. Date of publication February 27, 2012; date of current version March 7, 2013. Asterisk indicates corresponding author.

T. Sobayo, E. Gunnar, and C. Kazlauskas are with the Department of Biomedical Engineering, Illinois Institute of Technology, Chicago, IL 60616-3793 USA (e-mail: tsobayo@iit.edu; liz.gunnar@gmail.com; ckazlaus@iit.edu).

A. S. Fine was with the Department of Bioengineering at the University of Illinois, Chicago (UIC), IL 60607 USA. He is now with the Department of Neurology, Columbia University, New York, NY 10027 USA (e-mail: doctorfine1 @ gmail.com).

D. Nicholls is with the Department of Mathematics, Statistics and Computer Science, University of Illinois, Chicago (UIC), IL 60607 USA (e-mail: nicholls@math.uic.edu).

${ }^{*}$ D. J. Mogul is with the Department of Biomedical Engineering, Illinois Institute of Technology (IIT), Chicago, IL 60616-3793 USA (e-mail: mogul@iit.edu).

Color versions of one or more of the figures in this paper are available online at http://ieeexplore.ieee.org.

Digital Object Identifier 10.1109/TBME.2012.2189113 amplitude of surface electroencephalographic (EEG) recordings are commonly observed in patients undergoing epileptic seizures, research has long been aimed at obtaining a better understanding of the dynamics of brain electrophysiology as seizures evolve. Traditional dogma has asserted that pathologically synchronized activity may underlie seizures; however, recent studies have provided evidence that desynchronization in the brain may instead be an important component during seizures [2]-[4].

Currently, the dynamics by which seizures arise are not well understood. Some researchers have speculated that ictogenesis in focal epilepsies involve specific cortical and subcortical networks [5]-[7]. Recently, in the case of temporal lobe epilepsy (TLE), some researchers have postulated the multiple generator hypothesis. Multiple sites within the brain could act independently to initiate a seizure or drive another site into a seizure [8]. Further insights into the patterns of limbic seizure onset and termination would be immensely helpful in improving seizure control. The goal of this study was to explore the multisite spatiotemporal dynamics underlying seizure evolution in a kainic acid (KA) rat model of TLE using a novel analytical technique that allows for the nonlinear adaptive decomposition of electrophysiological signals into finite oscillatory components. TLE is a common form of focal epilepsy that is associated with seizure onset in one or more of the temporal limbic structures [9]. The circuit of Papez involving both hippocampi and the thalamus is one of the major pathways of the limbic system that it is believed to be involved in TLE [10] and hence it presented an important candidate for studying multisite network dynamics during seizures.

Local field potentials (LFPs) were recorded from electrodes implanted in three subcortical nuclei: the focal hippocampus (FH), the anteromedial thalamus (T), and the contralateral hippocampus $(\mathrm{CH})$ in rats in which KA was focally injected into $\mathrm{FH}$ to induce recurrent seizures. Frequencies, power, and measures of phase synchronization between oscillators extracted from LFPs both within and between subcortical structures were calculated. Significant and distinctly different patterns of phase synchrony were observed during ictogenesis and natural seizure termination.

\section{METHODS}

\section{A. Animals}

Eight male Sprague-Dawley rats, 48-57 days old, and weighing approximately 225-280 gm were used in this study. 
Experiments were conducted in accordance with the National Institutes of Health for the care and use of laboratory animals.

\section{B. Surgery}

Rats were anesthetized by a mixture of Ketamine $(80 \mathrm{mg} / \mathrm{kg})$ and Xylazine $(10 \mathrm{mg} / \mathrm{kg})$ delivered intraperitoneally. All procedures were performed in a Kopf stereotactic frame (KOPF Model 900, CA). Stereotactic targets were calculated using a stereotactic rat brain atlas [11]. Lambda, Bregma, and Sagittal sutures were used as landmarks to navigate to the desired stereotactic points. The skull was perforated using a high-speed stereotactic drill (Micromotor Drill, Stoelting Co, IL) with 1.22-mm diameter drill tips. Seven small burr holes were drilled: four were for the positioning of anchor screws and three for the placement of electrodes. Bipolar electrodes surrounding a single stainless steel injection cannula in one integrated electrode assembly (C315G-MS 303: PlasticsOne, Roanoke, VA) were stereotactically implanted into the CA3 region of the left hippocampus (-3.5-mm Bregma, 2.8-mm lateral, 3.7-mm deep). Bipolar recording electrodes (without cannula) were implanted into the $\mathrm{CH}$ (-3.5-mm Bregma, -2.8-mm lateral, 3.7-mm deep) and anteromedial thalamus (-1.8- $\mathrm{mm}$ Bregma, 0.3-mm lateral, 6.1-mm deep). The electrodes were then fixed to the screws and the skull using acrylic dental cement.

\section{Method and Analysis of EEG Recordings}

Following surgery, intracranial EEG signals were continuously recorded at a sampling rate of $2 \mathrm{kHz}$. Each experiment involved recording one half-hour of baseline activity followed by the injection of 3-5 nmol KA into the CA3 region of the left hippocampus to induce epileptogenesis. After injection, the internal cannula insert was withdrawn and a stainless steel insert was threaded through the cannula to provide one side of the recording pair. The reference electrode used was the skull stabilization screw most proximal to the electrode assembly. The raw signals from each of four recorded channels (namely intracranial recordings from the left and the right hippocampii and the anteromedial nucleus of thalamus as well as a subdural electrode covering the hemisphere contralateral to epileptogenic chemical application to provide surface EEG) were decomposed into a series of intrinsic mode functions (IMFs) using the method of empirical mode decomposition (EMD). The EMD can be characterized as an adaptive, nonlinear decomposition that results in a series of IMFs that together comprise the underlying oscillations (or basis functions) within a dataset [12], [13]. The basis functions are determined from the dynamics of the signal itself, which may be nonlinear and/or nonstationary [14]. After a series of intrinsic oscillators is obtained in this way, the instantaneous phase was calculated using the Hilbert analytic signal method [15]. This method is appropriate in this case because the IMFs represent narrowband signals. Unfiltered, multicomponent signals will yield a trajectory in the complex plane that has multiple centers of rotation. For an unambiguous determination of phase, the complex trajectory of the analytic signal must have only a single center of rotation, which the EMD technique provides.
Once the IMFs were obtained from each channel within a data segment, the strength of the relationships between the oscillators was determined. To accomplish this, the mean phase coherence [16] was calculated to obtain a square symmetric matrix relating the phase of each IMF oscillator obtained from EMD for each 5-s window of data. This matrix of phase relationships was then treated to eigenvalue decomposition. This method [17] allows one to extend a bivariate measure of phase coherence into a multivariate measure thereby permitting measures of synchrony across multiple oscillators. Essentially, this decomposition compares the strength of phase relationships between oscillators and clusters them according to mean fields. For any eigenvalue-eigenvector pair, a phase correlation value may be assigned as the strength of the connection of a given eigenvalue (unique for a given IMF) and an eigenvector (unique for the entire set of IMFs obtained from all channels). Furthermore, each eigenvalue is ordered, with the largest eigenvalue representing the most strongly correlated cluster with the participation of each oscillator in a given cluster quantified by the value of the eigenvector. Those eigenvalues above 1 are considered significant and the components of their eigenvectors identify participation in the corresponding cluster. In the analysis of our data, only the most significant clusters were analyzed to determine how synchrony dynamics evolve during seizures

\section{Analysis of Phase Synchrony}

Given an ensemble of coupled oscillators, in order to analyze the group's collective dynamics and, in particular, to describe synchronization processes, it is convenient to introduce the complex cluster variable $Z$ :

$$
Z=r e^{i \varphi}=\frac{1}{N} \sum_{j=1}^{N} e^{i \phi_{j}}
$$

where $\Phi_{j}$ is the phase of the $j$ th oscillator and $N$ is the number of oscillators [18]-[20]. The quantities $r$ and $\varphi$ denote the cluster amplitude and cluster phase at time $t$, respectively. The variable $r$ is also known as mean phase coherence [16] and phase amplitude coherence [21]. The cluster amplitude varies between 0 and 1 . As the ensemble becomes more synchronized, cluster amplitude increases toward unity. The difference in instantaneous cluster amplitude $\Delta r$ was calculated for the length of the entire recording. The variance of $\Delta r$ (or the phase amplitude dispersion) was then calculated using a 100-ms moving window. All the IMFs extracted from the same recording channel were analyzed using this measure in order to access the level of synchrony within a recording channel. As the oscillators become more coherent in phase, the phase amplitude dispersion should decrease.

In order to quantify the level of synchrony across recording channels, we employed the phase locking value (PLV) approach [22], [23]. For two signals $x(t)$ and $y(t)$ of equal time length with instantaneous phases $\Phi_{x}(t)$ and $\Phi_{y}(t)$, respectively, the PLV 
bivariate metric is given by

$$
\mathrm{PLV}=\left|\frac{1}{N} \sum_{j=1}^{N} e\left(i\left(\phi_{x}(j \Delta t)-\phi_{y}(j \Delta t)\right)\right)\right|
$$

where $\Delta t$ is the sampling period and $N$ is the number of points in the sampling period of each signal. PLV can vary between 0 and 1 where 1 indicates perfect phase synchronization and 0 indicates no phase synchronization.

For any two different recording channels, the PLVs of multiple pairs of IMFs that reside in the topmost significant cluster were used to construct a PLV matrix. The Euclidean norm (L2 norm) of the columns of the PLV matrix was then taken as a representative of phase synchrony between the channels. This operation reduced the PLV matrix from an $m \times n$ matrix to a $1 \times n$ matrix. In order to assess the significance of synchrony as quantified by PLV, we employed the use of surrogate datasets. Since the method by which surrogates are generated contribute to the accuracy of detection of meaningful synchrony, surrogates were initially produced using both the phase randomization technique and the more robust Fourier shuffling technique. Our tests on preliminary data showed that both surrogates were giving virtually the same results. We settled on using phaserandomized surrogates because it was computationally faster to generate these, which is consistent with our goal of eventually providing real-time analysis capabilities. Twenty surrogate datasets were created for each original data series. The mean and standard deviation for PLVs from the analysis of the surrogate data sets were used to calculate a threshold for $95 \%$ significance. Pairs of IMFs from the original data series possessing PLVs that exceeded the corresponding threshold were considered to have statistically significant synchrony.

\section{RESULTS}

\section{A. Intracranial Seizure Activity Elicited by Hippocampal Microinjection of $K A$}

LFPs were recorded in the anesthetized rat from three locations implicated in limbic seizures as described in Section II. Recordings showing $100 \mathrm{~s}$ of electrographic activity at each of the three intracranial electrode sites prior to (baseline) KA injection and a KA-induced spontaneous seizure are displayed in Fig. 1, panels A1 and A2, respectively. High amplitude LFPs were observed spontaneously occurring within $20 \mathrm{~min}$ after microinjection of KA $(20 \mu \mathrm{L})$ into the CA3 region of the $\mathrm{FH}$. In order to clearly identify the ictal period, the Teager energy (TE) [24], [25] of each LFP time series was calculated for either baseline (panel B1) or KA-induced spontaneous seizure (panel B2). The TE amplitude was found to be a reliable indicator of the ictal region that provided temporal markers for seizure onset and termination because high amplitude TE was only present when there was high amplitude LFP recordings correlated with KA-induced seizure activity. A threshold of the mean of the TE plus five times the standard deviation was used as criteria for selecting valid seizure activity. Since seizures are generally thought to represent aberrantly synchronized neural activity, we sought to develop a set of algorithms to accurately access and
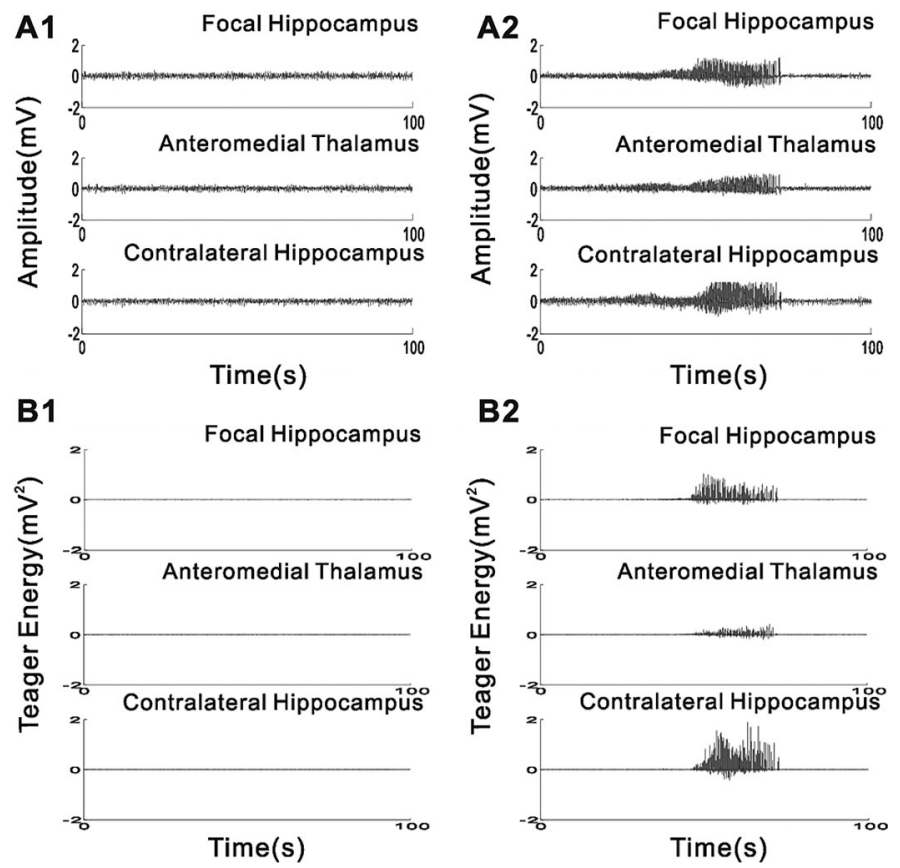

Fig. 1. Intracranial electrographic activity before and after KA injection. (A1) Baseline recordings of electrographic activity recorded from intracranial electrodes placed in the $\mathrm{FH}$, anteromedial thalamus, and $\mathrm{CH}$. The $F H$ is the left hippocampus in which the electrode assembly contains a microcannula for KA injection. (A2) Recordings of electrographic activity from the same three sites as in panel $\mathrm{A} 1$ but showing evidence of a spontaneous seizure induced by a prior injection of KA. TE of baseline recordings (B1) and of ictal recording (B2) gives a clear indication of the approximate time course of a seizure.

quantify this synchrony. EMD was used to decompose the raw time series signals into a finite collection of oscillators known as IMFs. The linear summation of the IMFs produces the original time series signal. EMD was chosen over other techniques such as Fourier for several reasons, most notably: 1) because it decomposes a time series into a set of orthogonal oscillators without assuming either linearity or stationarity of the underlying system responsible for the signal, and 2) because it produces a finite number of oscillators with clearly defined phase information that permits the rapid assessment of phase synchrony of complex intracranial electrophysiological time series.

\section{B. Dominant Oscillator Frequencies During Baseline Activity and Spontaneous Seizures}

The Hilbert analytic signal was constructed for each IMF in order to calculate the instantaneous phase for each. Moving averages of IMF frequencies were calculated by taking the absolute mean of the time derivative of the instantaneous phase of each IMF over 1-s intervals. In order to better visualize activity across a broad frequency range, the IMF frequencies are displayed in two separate plots: 1) high-frequency oscillators (defined as IMF's with a frequency $\geq 30 \mathrm{~Hz}$ ) and 2) low-frequency oscillators (IMF's with a maximum frequency $<30 \mathrm{~Hz}$ ). The frequency of $30 \mathrm{~Hz}$ was used as the differentiation between high- and low-frequency oscillators in part because many researchers [26]-[28] have reported that oscillators in the gamma band and above play an important role in epilepsy. Fig. 2(a) 

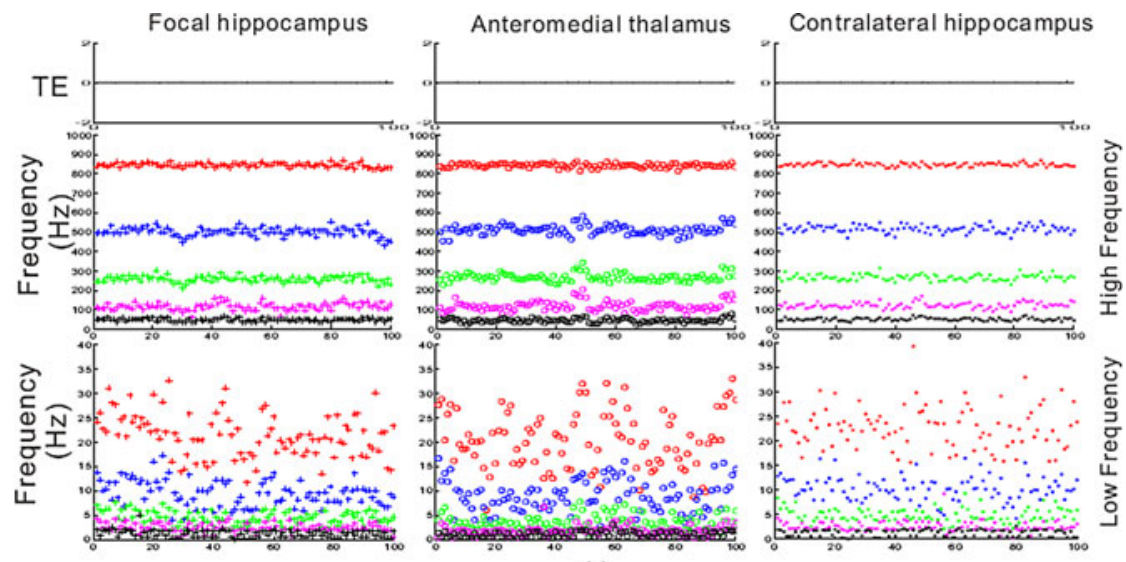

(a)

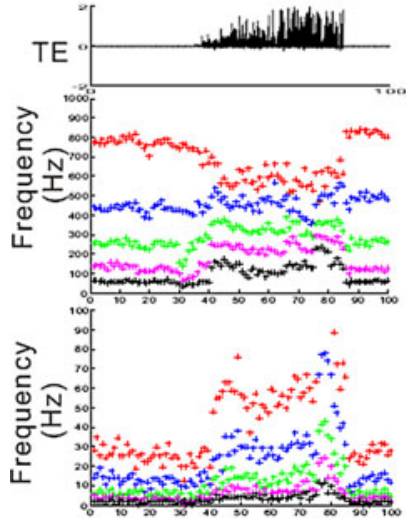

Time (s)

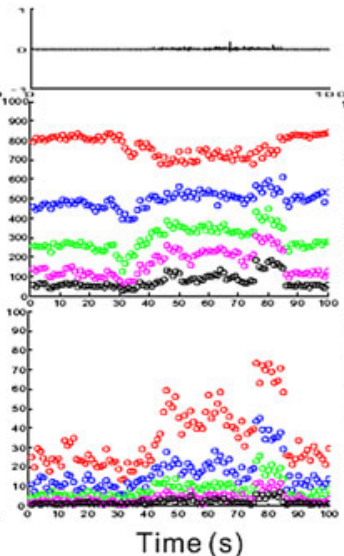

(b)

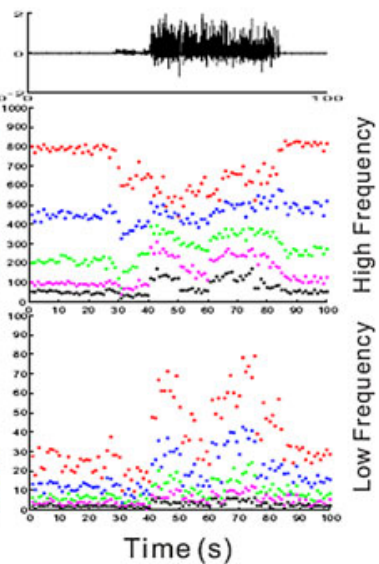

Fig. 2. Frequencies of IMF oscillators converge during a seizure. Frequency analysis was performed on the IMFs extracted from the electrographic signals recorded in the focal and contralateral hippocampi and anteromedial thalamus under: (a) Baseline conditions prior to KA exposure, and (b) during a spontaneous seizure following KA injection into the FH. For each time period of analysis, the TE is shown. Display of the analysis has been divided into the top five, high-frequency IMFs $(f>30 \mathrm{~Hz})$ and low-frequency IMFs $(f \leq 30 \mathrm{~Hz})$. Each IMF is color coded to the permit better visualization of oscillator dynamics over time. The distribution of frequencies was very stable under baseline conditions but during an ictal period, IMFs in both the high- and low-frequency categories tended toward convergence.

shows the frequencies extracted from the $\mathrm{FH}$, thalamus, and $\mathrm{CH}$ during a baseline recording from one of the experimental animals prior to KA injection. Fig. 2(b) shows the extracted IMF frequencies of the same animal in which KA-induced spontaneous seizure activity occurs. During the baseline, the frequency of oscillator activity remains relatively unchanged. However, at the onset of the ictal period (as indicated by significant increase in TE), there is a coincident drop in the frequencies of IMFs with preictal frequencies greater than $600 \mathrm{~Hz}$ while there is a rise in the frequencies of IMFs with preictal frequencies less than $600 \mathrm{~Hz}$. At the end of the ictal period, the frequencies of the IMFs return to their preictal values. This convergence of IMF frequencies was reflected by the large drop in the variance of the frequencies during the ictal period that was never present during baseline recordings.

\section{Power of IMF Oscillators Increases Significantly During Seizures}

In order to better assess the nature of the synchrony occurring during a seizure, the power of the IMFs extracted from each recording site was calculated in order to determine if the high energy activity seen during the ictal period could be attributed to an increase in the power of individual IMFs as opposed to simply an increase in IMF synchrony. The instantaneous power of an IMF was calculated by taking the square of its amplitude. The average power was then calculated by taking the mean of the instantaneous power over a 1-s interval. A comparison of the logarithm of the average power for the first 10 IMFs across all post-KA injection recordings ( $n=23$ seizures) reveal a significant increase in power during the ictal period (oneway ANOVA; $p<10^{-5}$ ). Fig. 3 shows the grand averages of the logarithm of the average power for these IMFs from the $\mathrm{FH}$, thalamus, and $\mathrm{CH}$ for all post-KA injection seizure recordings. The mean power during the ictal period ranges from approximately 5 to 8 times the mean power during the preictal or postictal periods indicating that at least some component of overall increase in seizure energy is due to increases in oscillator amplitudes at each of the three different structures.

\section{Phase Synchrony Assessed Within Recording Sites}

The Hilbert analytic signal was constructed for each IMF in order to calculate the instantaneous phase for each oscillator. 


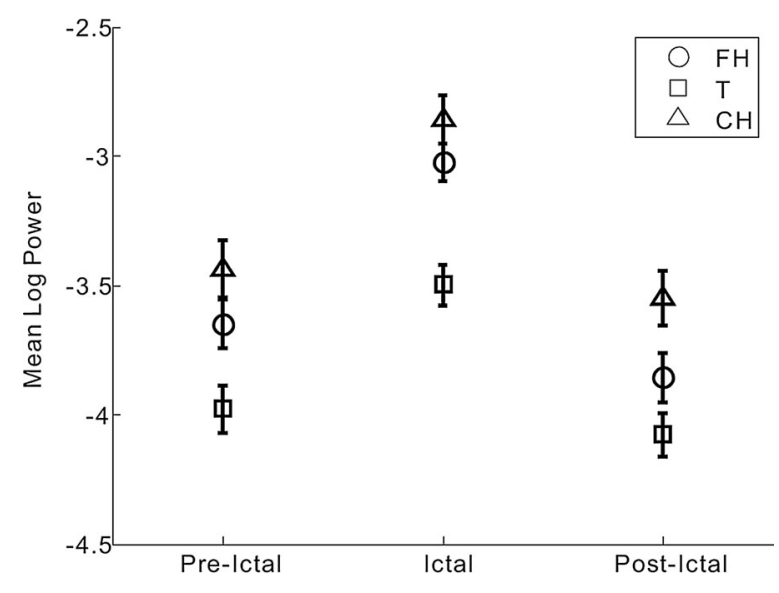

Fig. 3. Increase in IMF power observed during seizures. The average power of the first 10 IMFs from each of the three structures being recorded in 23 seizures. The results of all data were averaged within groups as preictal, ictal, and postictal periods based on levels of TE. ANOVA analysis indicated significant increase in power during the ictal period.

The instantaneous phases for all IMFs that belong to the same recording site were then used to calculate phase amplitude dispersions $(\operatorname{var}[\Delta r])$ as a way of quantifying phase synchrony within each recording site in the brain. In order to compare how synchrony evolved, five temporal categories for each seizure event in all post-KA injection recordings were created based on evolution of the Teager energy: namely, preictal, initiation, midseizure, termination, and postictal. Fig. 4(a)-(c) shows the phase amplitude dispersion for the $\mathrm{FH}$, thalamus, and $\mathrm{CH}$, respectively. Phase amplitude dispersion significantly decreased in all three recording sites (ANOVA; $p<10^{-4}$ for all three sites) as the middle of the seizure period was approached followed by an increase as seizures evolved toward termination. This pattern of dynamics is consistent with an overall increase in the synchrony of electrophysiological activity within each structure during limbic seizures and is, thus, consistent with seizures as hypersynchronous events.

\section{E. Phase Synchrony Assessed Between Recording Sites}

The ability to identify discrete oscillators in the electrophysiological behavior recorded in each brain structure permitted an assessment of how interregional synchrony evolved during the different phases of activity in an epileptic rat. In order to better understand the spatiotemporal dynamics occurring during a seizure, synchrony across recording sites was evaluated. To accomplish this, the PLV between pairs of sites was calculated. IMFs from all the recording sites were clustered to find groups of two or more oscillators whose dynamic behavior may be correlated. Significantly synchronized clusters were identified through eigenvalue decomposition as described in Section II. The top (or most) significantly synchronized clusters were identified and PLVs were calculated for pairs of IMFs based on three groupings: 1) IMFs from FH versus thalamus, 2) IMFs from thalamus versu. $\mathrm{CH}$, and 3) IMFs from $\mathrm{FH}$ versu. $\mathrm{CH}$. In order to assess the statistical significance of phase locking events, surrogates (as described in Section II) were employed.
Fig. 5 is a plot of the patterns of phase locking across pairs of structures for a post-KA injection recording containing a seizure. The PLVs for the different combination of pairs of recording site for all seizures were analyzed to identify continuous time periods where the phase locking exceeded $95 \%$ significance. Time periods with a minimum length of time of $100 \mathrm{~ms}$ were selected and the frequencies of both oscillators were identified. A threshold of $100 \mathrm{~ms}$ was used for the length of phase locking because it is longer than the typical time course that has been observed for excitatory postsynaptic currents in rat hippocampal neurons [29], [30]. In all of the seizures, there was significant phase locking (defined as a PLV that exceeded the 95\% significance threshold derived from surrogates for at least $100 \mathrm{msec}$ ) between the FH and the thalamus in the run up to the ictal activity. There are fewer occurrences of significant phase locking during the seizure; and then there is re-emergence of significant phase locking at the termination of the ictal activity. The pattern of significant phase locking between the FH and the $\mathrm{CH}$ and between the thalamus and $\mathrm{CH}$ varies considerably from seizure to seizure.

The result of this analysis for typical seizure events from the same animal is shown in Fig. 6. A synchrony analysis of activity during a segment of the baseline period before KA injection is shown in Fig. 6(a). TE is shown for each of the three recording periods in order to differentiate baseline from preictal or ictal activity. As in Fig. 2, analytical results have been divided into low-frequency $(f \leq 30 \mathrm{~Hz})$ and high-frequency $(f>30 \mathrm{~Hz})$ plots. Note that only very low-frequency synchrony $(f<10 \mathrm{~Hz})$ was evident in a nonseizing anesthetized animal prior to KA exposure. Panels B and C in Fig. 6 represent different spontaneous seizure events in the same animal. Low-frequency synchrony was observed throughout each recording period shown (see Fig. 6; panels B2 and C2) irrespective of seizure activity. Some higher frequency synchrony $(f \approx 25 \mathrm{~Hz})$ was sometimes seen but not reliably for all seizures in a given animal or between animals. However, more significant synchrony correlated closely to seizure activity was seen in the high-frequency range. For a majority of the seizures (21 out of 23), a 1:1 frequency locking in the $30-50 \mathrm{~Hz}$ frequency range was observed between all three sites and that almost always included the $\mathrm{CH}$ just prior to the beginning of the full-blown seizure as determined by the TE (panels B3 and C3). The frequencies of synchronization (mean $\pm \mathrm{SEM}$ ) in this run up to the seizure between the FH and the thalamus, the $\mathrm{FH}$ and the $\mathrm{CH}$, and the thalamus and the $\mathrm{CH}$ were $42.75 \pm 7.16,43.5 \pm 10$, and $38.04 \pm 5.35 \mathrm{~Hz}$, respectively. Because this synchrony appears prior to the highamplitude seizure onset, one might conclude that this dynamic is important to the generation of a seizure and not simply a consequence of it. In contrast, a frequency locking at $167.55 \pm$ $46.66 \mathrm{~Hz}$ (mean $\pm \mathrm{SEM}$ ) was observed primarily between the $\mathrm{FH}$ and the thalamus as the seizure naturally terminated. This dynamic was reliably observed across seizures within the same animal and between animals although differences in frequency of locking prior to termination were seen possibly because the epilepsies generated via KA injection may not have been precisely identical in underlying mechanisms. To our knowledge, this is the first evidence of clear interregional synchrony 
(a)

Focal Hippocampus

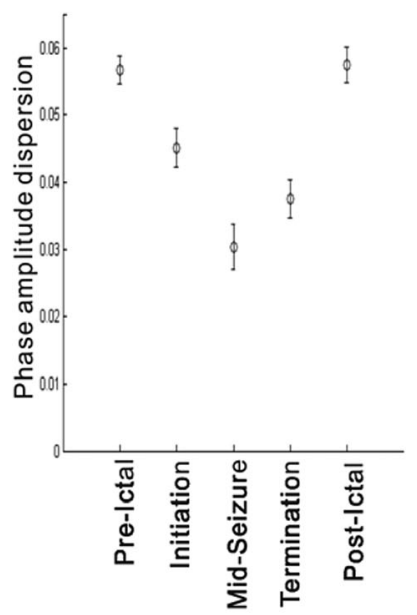

(b) Anteromedial Thalamus

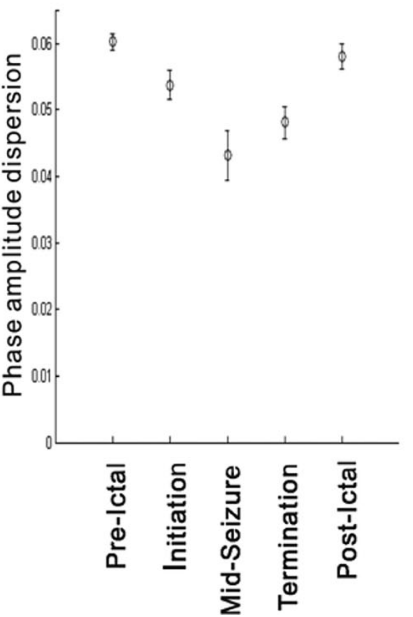

(c) Contralateral Hippocampus

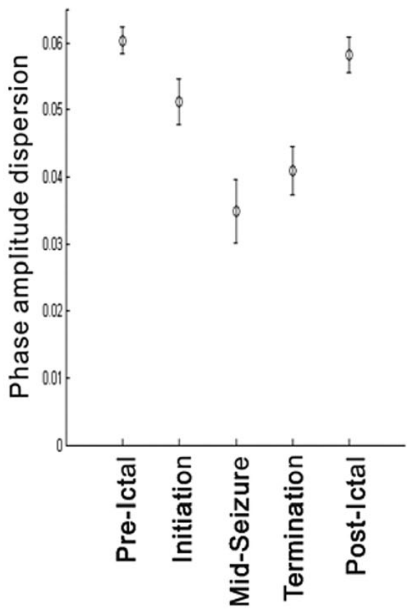

Fig. 4. Synchrony of oscillators measured within each structure increases as rats evolve into seizures. Average phase amplitude dispersion of all IMFs in (a) FH, (b) anteromedial thalamus, and (c) $\mathrm{CH}$ for 23 seizures. The results of all data were averaged within groups as preictal, initiation, midseizure, termination, and postictal periods. The phase amplitude dispersion achieved its lowest value during the middle of the seizure in all three brain structures.

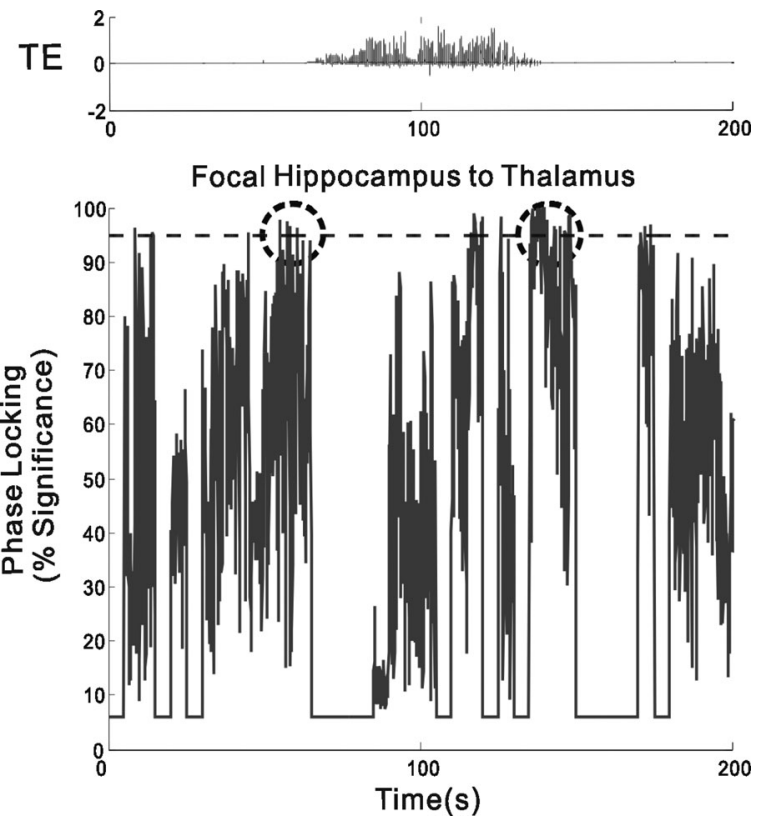

Fig. 5. Phase locking between pairs of structures indicates periods of phase synchrony during a seizure episode. Upper panel: TE indicates an ictal event containing one spontaneous seizure lasting approximately $70 \mathrm{~s}$. Paired comparisons of phase locking are shown between $\mathrm{FH}$ and thalamus. A dashed line denotes the $95 \%$ significance level of the PLV determined using randomized surrogates (see Methods). Dashed circles indicate periods of significant phase locking lasting longer than $100 \mathrm{~ms}$.

between these structures at well-defined frequencies and seizure states in an in vivo animal model of limbic epilepsy.

\section{DISCUSSION}

The aim of this study was to investigate the pattern of spatiotemporal dynamics that underlie spontaneous ictal activity during seizure evolution in the KA rat model of TLE. Under- standing how these dynamics evolve can provide not only a greater overall description of the electrophysiological mechanisms underlying seizures but may also yield important insights crucial to clinical management of the disease. Current diagnostic protocols as part of presurgical work-up for patients undergoing ablation therapy for disruption of ictogenesis could be greatly assisted by better understanding how structures in the brain interact with a putative seizure focus to induce seizure initiation. Such understanding of the dynamics might also provide important new insights for more accurate seizure prediction. Furthermore, better understanding of how brain circuitry interacts during the natural termination of a seizure may also provide key insights as to how exogenous manipulation of brain states such as through electrical stimulation might achieve greater efficacy. The method of EMD was employed in our analysis because it is an adaptive decomposition that does not require assumptions of linearity or stationarity of the dataset unlike Fourier analysis. Another advantage to using EMD is that it decomposes the original signal into a finite set of oscillatory components known as the IMFs with well-defined centers of rotation that provides us with specific dynamic targets of activity to both analyze for changes as a consequence of the disease as well as for potential disruption.

The frequencies of the IMF oscillators tended toward convergence during ictal events. A decrease in the frequency of very high-frequency $(f>600 \mathrm{~Hz}) \mathrm{IMFs}$ along with increases in the frequency of low-frequency IMFs were observed. Similar increases in the frequency of low-frequency oscillators $(f<60 \mathrm{~Hz})$ during a seizure have been reported using different analysis techniques [31]. Furthermore, an increase in the power of all IMFs during the ictal period was observed when compared to the preictal and postictal period suggesting that to the extent that these oscillators represent regional neuronal activity, seizures brought about increased neuronal activity and not just increased synchrony. Synchrony between 
A

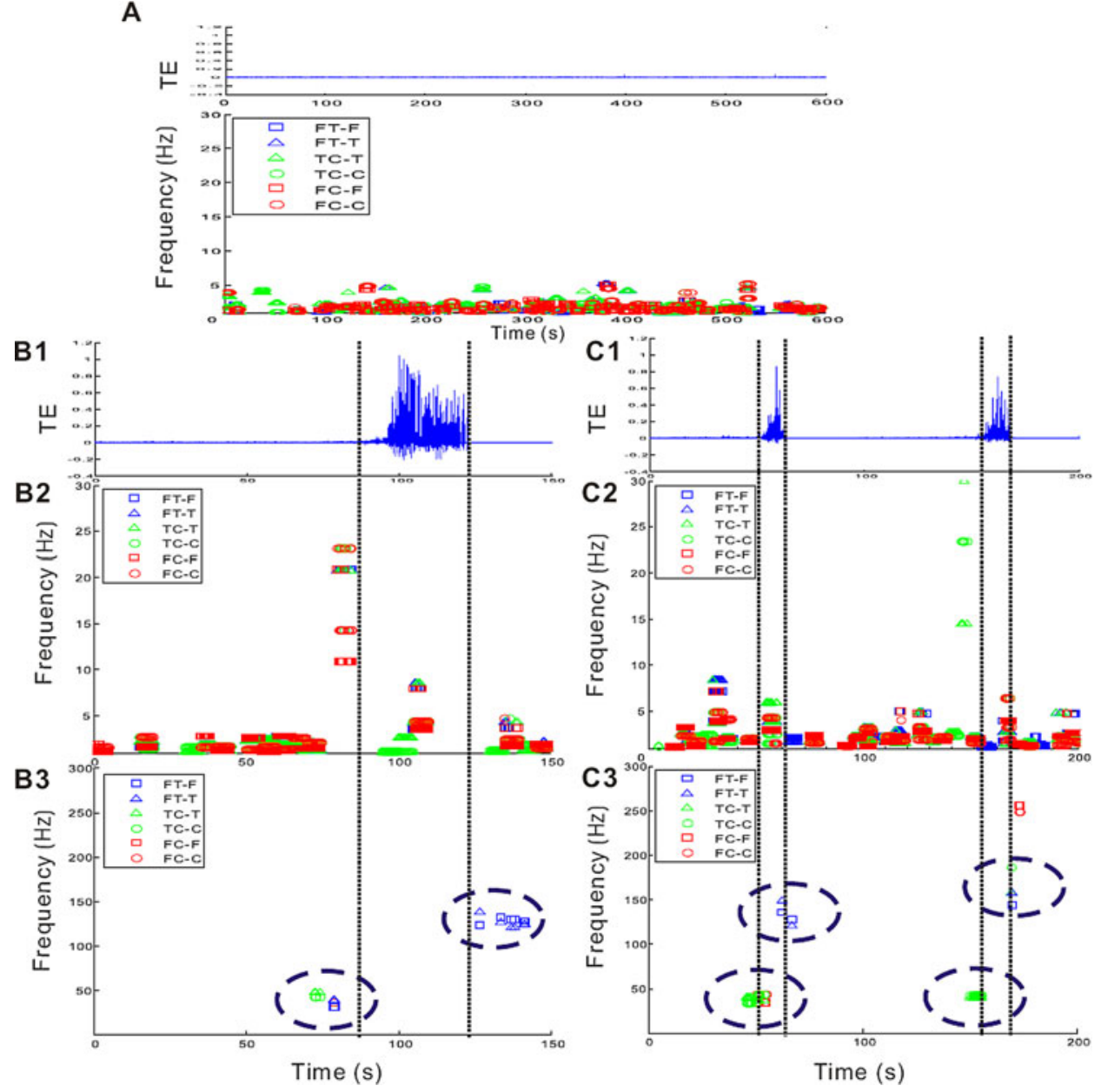

Fig. 6. Synchrony dynamics of oscillators in different structures in the rat brain exhibit phase locking (PL) as spontaneous seizures evolve. Synchrony dynamics are shown during three ictal periods in the same animal: (A) Baseline conditions prior to exposure to KA, (B) During a single spontaneous seizure, and (C) During a period in which two spontaneous seizures consecutively appeared. The TE of the FH electrical activity is provided at the top of each panel to delineate full-blown seizures from nonictal activity. Dashed lines indicate approximate time of seizure onset and seizure termination. Plots show periods of significant phase locking (i.e., $>95 \%$ ) that occur between pairs of brain structures for at least a contiguous $100 \mathrm{~ms}$. Because phase locking does not always occur 1:1, the frequency of IMF from each pair is shown (e.g., FT-F indicates significant phase locking between FH $(F)$ and thalamus $(T)$ with the FH IMF frequency indicated by the symbol noted in the inset key in each frequency plot. Low-frequency phase-locking among IMFs during a 10-min segment of baseline recording is shown in panel A. High frequency $(f>30 \mathrm{~Hz}) \mathrm{PL}$ is not shown here because none appeared in any of the experiments under pre-KA exposure baseline conditions. In this experiment, PL was only seen at frequencies below $10 \mathrm{~Hz}$ which was typical. Similar plots of low-frequency locking events as in panel A is shown in B2 and C2. As shown, PL prior to or coincident with seizure initiation occurred at $10 \mathrm{~Hz}<f<25 \mathrm{~Hz}$ although this was not reliably observed within the same seizing animal or between animals. In contrast, significant PL was reliably seen at higher frequencies coincident with seizure onset or termination (panels B3 and C3). PL events are highlighted by the dashed ovals. During ictogenesis, PL at $\sim 50 \mathrm{~Hz}$ was frequently observed that did not persist once full-blown seizure onset occurred. Furthermore, natural seizure termination was reliably coincident with seizure locking at frequencies between approximately $130-160 \mathrm{~Hz}$.

oscillators from within the same subcortical nucleus as well as synchrony between oscillators from pairs of different subcortical nuclei in the seizure network were calculated. Seemingly conflicting results were obtained when we examined the synchrony between the oscillators. It was found that the synchrony between oscillators extracted from the same recording sites increased during a seizure while oscillators from separate recording sites displayed significant and persistent synchronization at seizure initiation and termination. This suggests that epileptic seizures are characterized by increasing synchrony within each subcortical nucleus; however, there is reduced synchrony across the seizure circuit. This result is very similar to what has been shown about the difference in local and distant phase synchronization in generalized seizures in humans [32]. This also supports the idea that synchro- nization in the brain differs based on the spatial scale one is considering.

In Fig. 7, characteristic patterns that emerged during 10-s windows at the start of the ictal activity and as seizures naturally terminated are diagrammed. The network synchronization that is coincident with seizure termination is interesting in that it may offer insight into how to design and implement seizure perturbation protocols such as electrical deep brain stimulation aimed at seizure disruption. Three different patterns were observed as the seizures began to terminate: 1 ) over half of the seizures display locking between the $\mathrm{FH}$ and the thalamus (65\%); 2) 6\% display locking initially between the thalamus and the $\mathrm{CH}$; and 3) 29\% display locking between the $\mathrm{FH}$ and the $\mathrm{CH}$. By the end of the 10-s window, all of the seizures show direct or indirect phase locking between the FH and the thalamus. Our study indicates 
that there is an involvement of the thalamus in the evolution of partial seizures in TLE. This is in agreement with other animal studies. The thalamus has been shown to be involved during the course of seizures [33] and may play a crucial role in seizure development [34]. In addition, the study of human TLE patients by Guye et al. [35] showed that synchronization of signals from the thalamus and the temporal lobe structures occurs during seizures and tends to be particularly constant and marked in the last phase of the seizure.

Both the thalamus and the hippocampus have been used as targets for brain stimulation as treatment for intractable, medication-resistant focal epilepsy [36]. However, trials have only looked at single site stimulation at one of both structures and they have yielded inconsistent results. Furthermore, increasing synchronization in the brain has recently been proposed to be one possible way of terminating seizures [37]. These observations along with the findings of our study suggest that an electrical stimulation paradigm involving simultaneous stimulation of both the $\mathrm{FH}$ and the thalamus might be more effective in limbic seizure disruption than one that involves stimulation at only the hippocampus or the thalamus. Fig. 7 displays recurrent patterns observed in the course of KA-induced epileptic seizures; namely, a pattern of relatively low-frequency gamma oscillations before epileptic seizures followed by highfrequency oscillation commensurate with seizure termination. The question remains as to what physiological mechanisms may underlie this behavior. A possible mechanism for the synchrony of gamma oscillations observed during seizure onset could be related to the relatively rapid action of KA on glutamatergic receptors. $\mathrm{KA}$ is an agonist for kainite receptors (KARs) that are widely expressed in the hippocampal area CA3. Activation of KARs has been shown to lead to an increased excitation of CA3 and generation of gamma oscillations in hippocampal slices [26]. In addition to its excitatory effects, the activation of KARs by KA is known to cause a more delayed depression of GABAergic synaptic transmission [38], [39]. Furthermore, some studies have shown that it is possible for a network of neurons to transition from gamma oscillations to burst activity during periods as a result of an increase in excitatory input and a decrease in GABAergic input to CA3 pyramidal neurons [40]. Thus, the high-frequency oscillations observed at seizure termination could be reflective of fast IPSPs [41] generated by inhibitory interneurons from outside the hippocampal CA3. Furthermore, these high-frequency oscillations may be at least partly manifested through rapid synaptic communication via gap junctions [42]. This electrophysiological effect has been hypothesized to be indicative of the underlying inhibitory mechanisms trying to reassert control of an overactive neural circuit.

It is unknown whether the IMFs from EMD represent actual physiological oscillators or merely depict the phase dynamics of one or more network oscillations. This may cause uncertainties with direct physical interpretation of the IMFs obtained from the decomposition although it is unlikely to interfere with synchrony analysis. However, more to the point is that the standard frequency analysis of brain electrographic activity using Fourier analysis has commonly produced analytical summaries of brain (a)

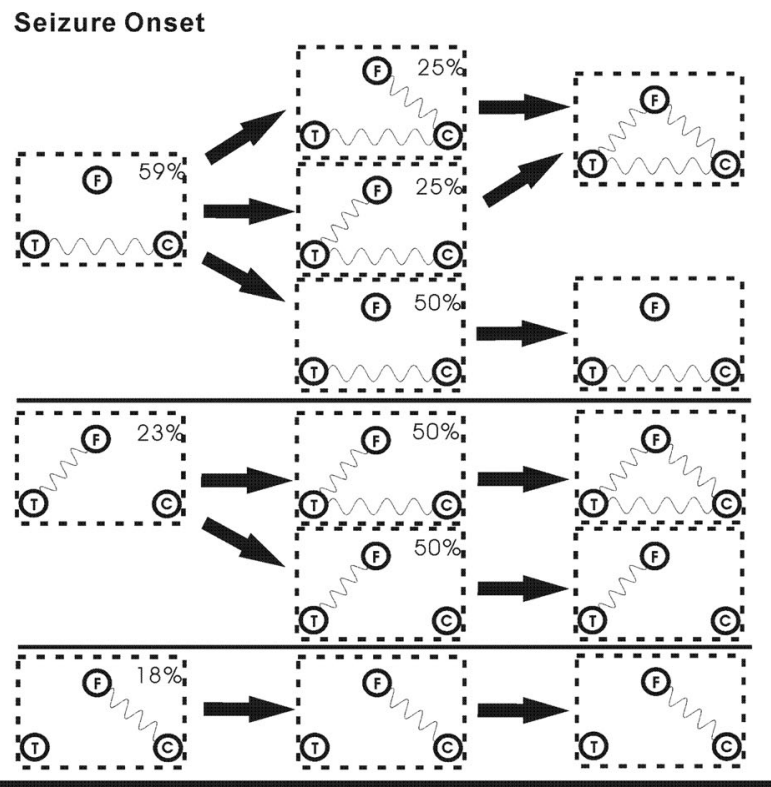

(b) Seizure Termination
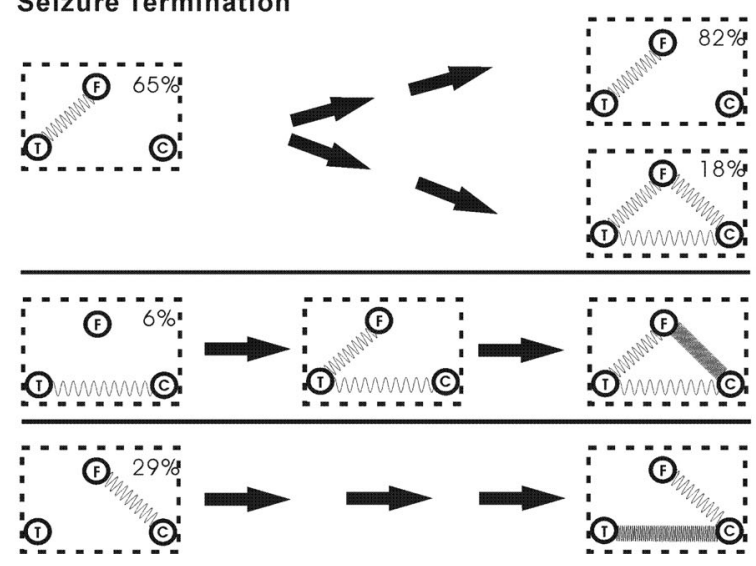

Fig. 7. Schematic of synchronization patterns between brain structure pairs that occurred coincident with seizure initiation or termination. The frequency of the line connecting any two subcortical nuclei represents the frequency range of the oscillators as defined here. Low: $30-100 \mathrm{~Hz}$; medium: 100-160 Hz; high: $>160 \mathrm{~Hz}$. The number in the boxes is the percentage of seizures depicting that particular pattern. $F, T$, and $C$ denote the $\mathrm{FH}$, thalamus, and $\mathrm{CH}$, respectively. (a) Depictions of the different patterns of significant and persistent phase locking between the three subcortical nuclei coincident with the start of ictal activity. In $82 \%$ of the seizures, phase locking occurred between the thalamus and one of the hippocampi in the low-frequency range as defined above. Four different permutations of phase locking occurred prior to the full-blown seizure after which no higher frequency synchrony was observed. (b) Depictions of the different patterns of significant and persistent phase locking between the three subcortical nuclei in a $10-\mathrm{s}$ window at the natural termination of ictal activity. At seizure termination, medium frequency synchrony was initially observed. This dynamic evolved to include higher frequency PL that sometimes included more regions than initially although it should be noted that, in $53 \%$ of the seizure termination incidents, synchrony occurred primarily between thalamus and $\mathrm{FH}$.

activity in frequency bands (i.e., theta, beta bands, etc.) whose physiological relevance is also not well understood but whose dynamics characterization has proved invaluable to understanding correlative relevance of brain behavior. Furthermore, most other decomposition methods, to our knowledge, depend on assumptions of some a priori determined oscillator waveforms underlying the time series signals that relies on assumptions of linearity or stationarity of neuronal network electrophysiology 
that may very well be erroneous. Thus, the analytical outcomes using the techniques described in this paper provided discrete oscillator phase and frequency information that offers new insights into intersite network behavior in the brain during seizures and provides specific spatiotemporal dynamics that may be directly manipulatable by exogenous electrical perturbation.

\section{ACKNOWLEDGMENT}

The authors T. Sobayo and A. S. Fine contributed equally to this research. All experiments were conducted in the Laboratory of D.J.M. at the Illinois Institute of Technology.

\section{REFERENCES}

[1] R. S. Fisher, W. van Emde Boas, W. Blume, C. Elger, P. Genton, P. Lee, and J. Engel, Jr., "Epileptic seizures and epilepsy: Definitions proposed by the international league against epilepsy (ILAE) and the international bureau for epilepsy (IBE)," Epilepsia, vol. 46, pp. 470-472, Apr. 2005.

[2] B. S. Gutkin, G. B. Ermentrout, and A. D. Reyes, "Phase-response curves give the responses of neurons to transient inputs," J. Neurophysiol., vol. 94, pp. 1623-1635, Aug. 2005.

[3] T. I. Netoff and S. J. Schiff, "Decreased neuronal synchronization during experimental seizures," J. Neurosci., vol. 22, pp. 7297-7307, Aug. 15, 2002.

[4] K. Schindler, H. Leung, C. E. Elger, and K. Lehnertz, "Assessing seizure dynamics by analysing the correlation structure of multichannel intracranial EEG," Brain, vol. 130, pp. 65-77, Jan. 2007.

[5] F. Bartolomei, F. Wendling, J. J. Bellanger, J. Regis, and P. Chauvel, "Neural networks involving the medial temporal structures in temporal lobe epilepsy," Clin. Neurophysiol., vol. 112, pp. 1746-1760, Sep. 2001.

[6] J. Gotman, "Epileptic networks studied with EEG-fMRI," Epilepsia, vol. 49 (Suppl. 3), pp. 42-51, 2008.

[7] S. S. Spencer, "Neural networks in human epilepsy: Evidence of and implications for treatment," Epilepsia, vol. 43, pp. 219-227, Mar. 2002.

[8] E. H. Bertram, "Temporal lobe epilepsy: Where do the seizures really begin?," Epilepsy Behav., vol. 14, Suppl. 1, pp. 32-37, 2009.

[9] S. S. Spencer and D. D. Spencer, "Entorhinal-hippocampal interactions in medial temporal lobe epilepsy," Epilepsia, vol. 35, pp. 721-727, Jul./Aug. 1994.

[10] H. Oikawa, M. Sasaki, Y. Tamakawa, and A. Kamei, "The circuit of Papez in mesial temporal sclerosis: MRI," Neuroradiology, vol. 43, pp. 205-210, Mar. 2001.

[11] G. Paxinos and C. Watson, The Rat Brain in Stereotaxic Coordinates, 5 ed. New York: Academic, 2004.

[12] N. E. Huang, Z. Shen, S. R. Long, M. C. Wu, H. H. Shih, Q. Zheng, N.-C. Yen, and H. H. Liu, "The empirical mode decomposition and the Hilbert spectrum for nonlinear and non-stationary time series analysis," Proc. Royal Soc. London, vol. 454, pp. 903-995, 1998.

[13] C. M. Sweeney-Reed and S. J. Nasuto, "A novel approach to the detection of synchronisation in EEG based on empirical mode decomposition," $J$. Comput. Neurosci., vol. 23, pp. 79-111, Aug. 2007.

[14] A. S. Fine, D. P. Nicholls, and D. J. Mogul, "Assessing instantaneous synchrony of nonlinear nonstationary oscillators in the brain," J. Neurosci. Methods, vol. 186, pp. 42-51, Jan. 30, 2010.

[15] D. Gabor, "Theory of communication," J. IEEE London, vol. 93, pp. 429$457,1946$.

[16] F. Mormann, K. Lehnertz, P. David, and C. E. Elger, "Mean phase coherence as a measure for phase synchronization and its application to the EEG of epilepsy patients," Physica D, vol. 144, pp. 358-369, 2000.

[17] C. Allefeld, M. Muller, and J. Kurths, "Eigenvalue decomposition as a generalized synchronization cluster analysis," Int. J. Bifurcation Chaos, vol. 17, pp. 3493-3497, 2007.

[18] Y. Kuramoto, Chemical Oscillations, Waves and Turbulence. Berlin: Springer-Verlag, 1984.

[19] P. A. Tass, Phase Resetting in Medicine and Biology: Stochastic Modelling and Data Analysis, Berlin; New York: Springer Verlag, 1999.

[20] A. T. Winfree, "Phase control of neural pacemakers," Science, vol. 197, pp. 761-763, Aug. 19, 1977.

[21] S. J. Schiff, T. Sauer, R. Kumar, and S. L. Weinstein, "Neuronal spatiotemporal pattern discrimination: The dynamical evolution of seizures," Neuroimage, vol. 28, pp. 1043-1055, Dec. 2005.
[22] J. P. Lachaux, E. Rodriguez, J. Martinerie, and F. J. Varela, "Measuring phase synchrony in brain signals," Hum Brain Mapp., vol. 8, pp. 194-208, 1999.

[23] V. Sakkalis, V. Tsiaras, M. Zervakis, and I. Tollis, "Optimal brain network synchrony visualization: Application in an alcoholism paradigm," in Proc. IEEE Eng. Med. Biol. Soc. Conf., 2007, pp. 4285-4288.

[24] A. B. Gardner, A. M. Krieger, G. Vachtsevanos, and B. Litt, "One-class novelty detection for seizure analysis from intracranial EEG," The $J$. Mach. Learning Res., vol. 7, pp. 1025-1044, 2006.

[25] H. P. Zaveri, W. J. Williams, and J. C. Sackellares, "Energy based detection of seizures," in Proc. 15th Annu. Int. Conf. IEEE Eng. Med. Biol., 1993, pp. 363-364.

[26] A. Fisahn, "Kainate receptors and rhythmic activity in neuronal networks: Hippocampal gamma oscillations as a tool," J. Physiol., vol. 562, pp. 6572, Jan. 1, 2005.

[27] R. Kohling, M. Vreugdenhil, E. Bracci, and J. G. Jefferys, "Ictal epileptiform activity is facilitated by hippocampal GABAA receptor-mediated oscillations," J. Neurosci., vol. 20, pp. 6820-6829, Sep. 15, 2000.

[28] M. J. Lehmkuhle, K. E. Thomson, P. Scheerlinck, W. Pouliot, B. Greger and F. E. Dudek, "A simple quantitative method for analyzing electrographic status epilepticus in rats," J. Neurophysiol., vol. 101, pp. 16601670, Mar. 2009

[29] M. Ivenshitz and M. Segal, "Simultaneous NMDA-dependent long-term potentiation of EPSCs and long-term depression of IPSCs in cultured rat hippocampal neurons," J. Neurosci., vol. 26, pp. 1199-210, Jan. 25, 2006.

[30] S. Mennerick and C. F. Zorumski, "Presynaptic influence on the time course of fast excitatory synaptic currents in cultured hippocampal cells," J. Neurosci., vol. 15, pp. 3178-3192, Apr. 1995.

[31] D. Hsu, M. Hsu, H. L. Grabenstatter, G. A. Worrell, and T. P. Sutula, "Timefrequency analysis using damped-oscillator pseudo-wavelets: Application to electrophysiological recordings," J. Neurosci. Methods., vol. 194, pp. 179-192, Dec. 15, 2010.

[32] L. Garcia Dominguez, R. A. Wennberg, W. Gaetz, D. Cheyne, O. C. Snead, 3rd, and J. L. Perez Velazquez, "Enhanced synchrony in epileptiform activity? Local versus distant phase synchronization in generalized seizures," J. Neurosci., vol. 25, pp. 8077-8084, Aug. 31, 2005.

[33] E. H. Bertram, P. S. Mangan, D. Zhang, C. A. Scott, and J. M. Williamson, "The midline thalamus: Alterations and a potential role in limbic epilepsy," Epilepsia, vol. 42, pp. 967-978, Aug. 2001.

[34] R. M. Cassidy and K. Gale, "Mediodorsal thalamus plays a critical role in the development of limbic motor seizures," J. Neurosci., vol. 18, pp. 9002-9009, Nov. 1, 1998.

[35] M. Guye, J. Regis, M. Tamura, F. Wendling, A. McGonigal, P. Chauvel, and F. Bartolomei, "The role of corticothalamic coupling in human temporal lobe epilepsy," Brain, vol. 129, pp. 1917-1928, Jul. 2006.

[36] B. C. Jobst, T. M. Darcey, V. M. Thadani, and D. W. Roberts, "Brain stimulation for the treatment of epilepsy," Epilepsia, vol. 51 (Suppl. 3), pp. 88-92, Jul. 2010.

[37] K. Schindler, C. E. Elger, and K. Lehnertz, "Increasing synchronization may promote seizure termination: Evidence from status epilepticus," Clin. Neurophysiol., vol. 118, pp. 1955-1968, Sep. 2007.

[38] R. S. Fisher, "Electrophysiologicl mechanisms of kainic acid-induced epileptiform acitivity in the rat hippocampal slice," J. Neurosci., vol. 4, pp. 1312-1323, May 1984.

[39] J. Lourenco, I. Matias, G. Marsicano, and C. Mulle, "Pharmacological activation of kainate receptors drives endocannabinoid mobilization," $J$. Neurosci., vol. 31, pp. 3243-3248, Mar. 2, 2011.

[40] R. D. Traub, I. Pais, A. Bibbig, F. E. Lebeau, E. H. Buhl, H. Garner, H. Monyer, and M. A. Whittington, "Transient depression of excitatory synapses on interneurons contributes to epileptiform bursts during gamma oscillations in the mouse hippocampal slice," J. Neurophysiol., vol. 94, pp. 1225-1235, Aug. 2005.

[41] A. Bragin, J. Engel, Jr., C. L. Wilson, I. Fried, and G. Buzsaki, "Highfrequency oscillations in human brain," Hippocampus, vol. 9, pp. 137$142,1999$.

[42] N. Maier, M. Guldenagel, G. Sohl, H. Siegmund, K. Willecke, and A. Draguhn, "Reduction of high-frequency network oscillations (ripples) and pathological network discharges in hippocampal slices from connexin 36deficient mice," J. Physiol., vol. 541, pp. 521-528, Jun. 1, 2002.

Authors' photographs and biographies not available at the time of publication. 